\title{
A NEW HIGH DENSITY, HIGH PRECISION ASTROMETRIC CATALOG
}

\author{
M.I. ZACHARIAS ${ }^{1,2}$, G.L. WYCOFF ${ }^{2}$, G.G. DOUGLASS ${ }^{2}$, \\ T.E. CORBIN ${ }^{2}$ AND N. ZACHARIAS ${ }^{1,2}$ \\ ${ }^{1}$ Universities Space Research Association \\ ${ }^{2}$ U.S.Naval Observatory, Washington, DC, USA
}

\section{Introduction}

The Version 1.0 of the U.S. Naval Observatory Twin Astrographic Catalog (TAC) contains positions of 705,679 stars (Zacharias et al 1996). The TAC 1.0 covers over $90 \%$ of the sky from $+90^{\circ}$ to $-18^{\circ}$ declination; 260 out of 5180 plates are not yet measured. Plates were taken in both the yellow $(508-578 \mathrm{~nm})$ and in the blue $(410-486 \mathrm{~nm})$ bandpass between 1978 and 1986 at the U.S. Naval Observatory (USNO) in Washington, DC.

\section{Catalog Properties}

Preliminary photometry from these plate measures was obtained by using the Hipparcos Input Catalogue (HIC); 697,140 stars have a V and 675,800 stars a $\mathrm{B}$ magnitude. The limiting magnitudes are about $\mathrm{V}=11.5$ (Figure 1) and $B=12.0$. The formal standard error of a mean magnitude is 0.11 mag or better for $50 \%$ of the stars.

The International Reference Stars (IRS) catalog (FK5/J2000 system) was used as the reference star catalog for the astrometric reductions. Magnitude-dependent systematic errors were found and preliminarily corrected (Zacharias and Douglass 1995). The average precision of a catalog position is 90 mas per coordinate at the mean epoch of observation (Figure 2).

Proper motions are being obtained by combining the TAC 1.0 results with new reductions of the Astrographic Catalogue (AC, Urban et al. 1996), as the latter work progresses. The precision of these proper motions varies between 2 and 8 mas/yr (Figure 3) depending on the quality and epochs of the AC plates. 


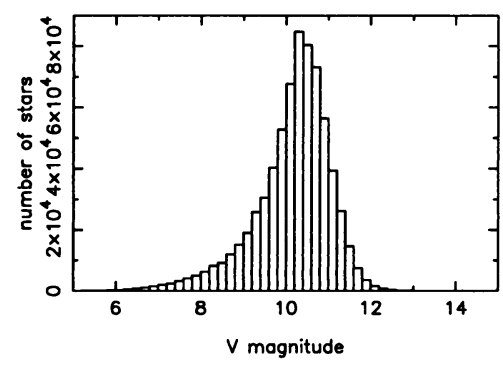

Figure 1. Distribution of $\mathrm{V}$ magnitudes of 697,140 TAC 1.0 stars from the yellow lens plates

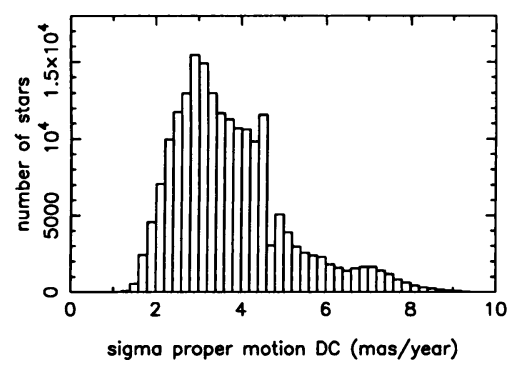

Figure 3. Estimated errors of declination proper motions for 195,622 stars of $3 \mathrm{AC}$ test zones

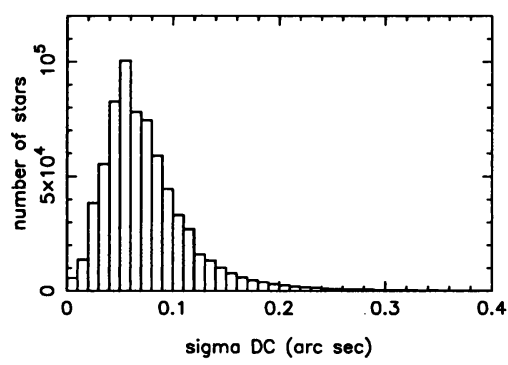

Figure 2. Mean positional errors in $\delta$ (mean epochs of observation) for 692,133 stars with at least 2 observations

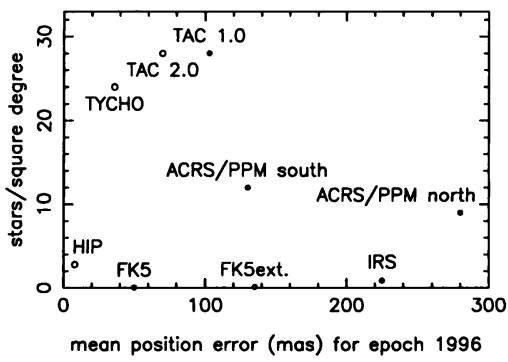

Figure 4. Star density vs. mean position error (epoch 1996) of astrometric catalogs (full = existing, open $=$ future data).

The TAC 1.0 is almost 3 times more precise than the PPM or ACRS in the northern hemisphere at current epochs (Figure 4) and the star density is about 3 times higher. The TAC 1.0 has a higher star density than the Tycho catalog and provides independent, high precision positions for a large fraction of the Tycho stars at an epoch about 10 years earlier than the Tycho mean epoch. The AC, TAC and Tycho data combined will give high quality proper motions for over half a million stars in 1997 . The catalog and details are at

$$
\text { aries .usno. navy. } \mathrm{mil} / \mathrm{ad} / \mathrm{tac} . \mathrm{html} \text {. }
$$

\section{References}

Urban, S.E., Martin, J.C., Jackson E.S., and Corbin, T.E. 1996 New Reductions of the Astrographic Catalogue:Plate adjustments of the Algiers, Oxford I and II, and Vatican Zones, Astron.Astrophys., 118, 163

Zacharias, M.I., and Douglass, G.G. 1995 Preliminary Results of the U.S. Naval Observatory Twin Astrographic Catalog (TAC) Plate Reductions, Bull.Am.Astron.Soc. 27, 857

Zacharias, N., Zacharias, M.I., Douglass, G.G., and Wycoff, G.L. 1996 The Twin Astrographic Catalog (TAC) Version 1.0, Astron.J., 112, 2336. 